\title{
Research Article \\ Research on Fault Diagnosis Method Based on Rule Base Neural Network
}

\author{
Zheng Ni, ${ }^{1}$ Zhang Lin, ${ }^{1}$ Wang Wenfeng, ${ }^{1,2,3}$ Zhang Bo, ${ }^{1}$ Liu Yongjin, ${ }^{1}$ and Zhang Dajiang ${ }^{1}$ \\ ${ }^{1}$ Air and Missile Defense College, Air Force Engineering University, Xi'an 710051, China \\ ${ }^{2}$ The 302ed Institute of the Tenth Academy of China Aerospace Science \& Industry Corporation, Guiyang, Guizhou 550002, China \\ ${ }^{3}$ School of Automation Science and Electrical Engineering, Beihang University, Beijing 100191, China
}

Correspondence should be addressed to Wang Wenfeng; wwf981612@163.com

Received 10 June 2016; Revised 15 September 2016; Accepted 16 October 2016; Published 12 January 2017

Academic Editor: Xiao He

Copyright (C) 2017 Zheng Ni et al. This is an open access article distributed under the Creative Commons Attribution License, which permits unrestricted use, distribution, and reproduction in any medium, provided the original work is properly cited.

\begin{abstract}
The relationship between fault phenomenon and fault cause is always nonlinear, which influences the accuracy of fault location. And neural network is effective in dealing with nonlinear problem. In order to improve the efficiency of uncertain fault diagnosis based on neural network, a neural network fault diagnosis method based on rule base is put forward. At first, the structure of BP neural network is built and the learning rule is given. Then, the rule base is built by fuzzy theory. An improved fuzzy neural construction model is designed, in which the calculated methods of node function and membership function are also given. Simulation results confirm the effectiveness of this method.
\end{abstract}

\section{Introduction}

In recent years, with the increasing development of science technology and automation, weapon equipment systems have been updated constantly. The fault occurrence probability is higher with the development tendency of large scale, complexity, and nonlinearity. The complexity of weapon system and the nonlinearity between fault cause and fault phenomenon reduce the diagnosis sensitivity of weapon system and improve the error report ratio, which increase the difficulty of fault diagnosis [1-4].

The BP neural network is the most extensive used neural network model. It not only has strong self-learning ability and self-organization, but also can process nonlinear problem. So BP neural network has a great superiority in processing nonlinear fault. Reference [5] uses the method of [6] to improve the $\mathrm{BP}$ algorithm of three-layer perceptron and apply it to the fault diagnosis of steam turbine, which optimizes the network step by step and accelerates the convergence speed of the net by this method. Reference [7] applies BP neural network into the fault diagnosis of gear in vehicle transmissions. Reference [8] constructs the fault diagnosis method of solid rocket motor based on fuzzy neural network, which combines BP network and fuzzy inference. Reference [9] synthesizes rule base, Bayesian belief network, and neural network into integrated software while fault prognosis. Reference [10] promotes an intelligent fault diagnosis expert system based on rule base, which merges together with fuzzy theory and enhances the transparence of fault diagnosis.

While ensuring learning samples, traditional rule base relies on expert experience and knowledge mainly and manifests by IF-THEN formation. A formal produced rule can manifest the fault whose causality is distinct, but it cannot manifest the fault whose causality is not distinct and relationship between phenomenon and reason is nonlinear. Fuzzy theory has the ability to process unsure and inexact information well. So this paper combines fuzzy theory with neural network, applies fuzzy logic into the description of high-rise logic frame, and uses neural network to process data, which enhances the accuracy of fault ruling base and acquires exact training samples. The efficiency of fault diagnosis is improved by applying the method to fault diagnosis.

\section{Problem Description}

On the basis of known data and fault model, the neural network can acquire mapping relationship between data and fault model by the studying of samples. Neural network can 
acquire complete and precise message from the incomplete and unsafe ones and realize the analogue to human thinking model in aspect of signal processing and learning ability [9].

In the reality procedure, mean square error can be approximated by $G(x)$ :

$$
G(x)=(t-a)^{T}(t-a) .
$$

Among these, $x$ is weighted value $W$ or biasing $b, t$ is target output, and $a$ is realistic output.

The essence of the neural network training process is an adjusting process of weights $W$ and bias $b$ constantly. In order to guarantee the convergence of nervous system, the steepest method is usually used to adjust the weights $W$ and bias $b$ :

$$
\begin{aligned}
W^{m}(k+1) & =W^{m}(k)-\alpha(k) \times \frac{\partial G(x)}{\partial W^{m}} \\
& =W^{m}(k)-\alpha(k) \times \frac{\partial G(x)}{\partial n^{m}} \times\left(a^{m-1}\right)^{T}, \\
b^{m}(k+1) & =b^{m}(k)-\alpha(k) \times \frac{\partial G(x)}{\partial b^{m}} \\
& =b^{m}(k)-\alpha(k) \times \frac{\partial G(x)}{\partial n^{m}},
\end{aligned}
$$

where $k$ is the number of iterations and $\alpha$ is velocity of learning.

$G(x)$ is minimum or its value is small to a certain value $\varepsilon$ through continuous recursion and iteration. Due to the existence of the iterative process, the influence of original samples uncertainty to the final result of the fault diagnosis is enhanced. It also leads to the following problems in the process of application of neural network:

(1) The difficulty of acquiring samples: neural network needs a lot of samples training before fault diagnosis, but the presence of sample cannot be ensured and the sample distribution whether equal or not cannot be judged because of the random. It can hardly guarantee the training effect and the accuracy. At the same time, in actual engineering equipment, abundant and distributed equally fault samples are difficult to acquire [11].

(2) The underutilization of experienced knowledge: in the territory of fault diagnosis, the using of experienced knowledge is important. Some faults can be found the fault reasons by experienced knowledge. But neural network acquires experienced knowledge from learning samples and this experienced knowledge has great boundedness. So, the learning method of neural network reduces the accuracy of fault diagnosis.

We can see that the accuracy of fault diagnosis has an intimate relationship with the quality of training sample. Under the condition of unsure message, the paper applies fuzzy theory into neural network to deal with the related fault better and acquire more typical samples, which makes advantages of known experienced knowledge to realize a

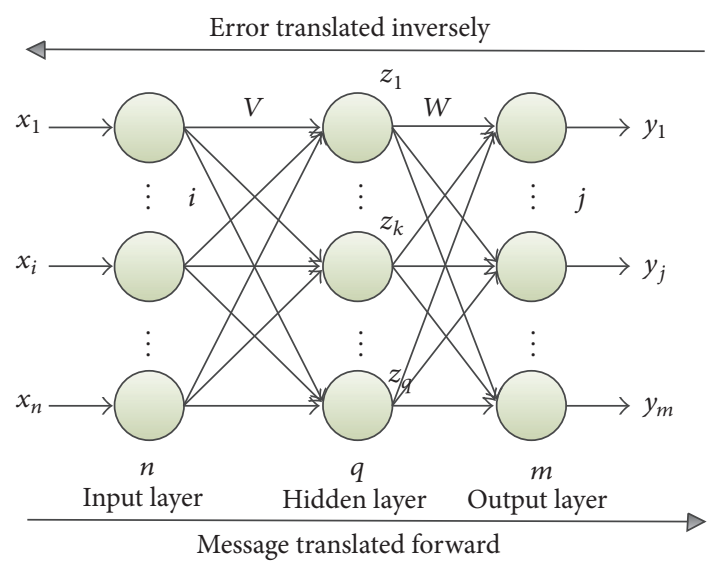

FIGURE 1: The structure of BP neural network.

precise location. Fuzzy theory is used to process unsure message to acquire a more objective fault rule base. Then the improved neural network is used to diagnose fault. The accuracy of fault diagnosis is improved.

\section{The Design of BP Neural Network Fault Diagnosis Model Based on Rule-Learning}

The general steps of fault diagnosis based on neural network include confirming network structure, then having learning training to network based on known fault phenomenon and reason, adjusting weight and threshold, and finally acquiring result of diagnosis using trained network and input fault phenomenon. So, the fault diagnosis method based on neural network can be divided into two main parts: BP neural network training and BP neural network fault diagnosis.

In order to improve the accuracy of fault diagnosis based on neural network, the belief rule base which is constructed by the fuzzy theory in the basis of existent experience knowledge is used to preprocess original samples before the neural network training. The processed samples are used to train the neural network. Finally, the trained neural network can be used to diagnose fault and obtain the fault diagnosis results. So the key to improve the neural network fault diagnosis is how to construct belief rule based on the fuzzy theory.

3.1. Structure of Classical Neural Network. From the angle of mapping, the procedure of diagnosis can be seen as a mapping from fault eigenvectors set to fault set. So the fault diagnosis is to find the mapping relationship between fault sign and reason. The neural network is to construct the fault mapping relationship through neural network structure. Continuous function of random closed interval can be approximated by three-layer BP neural networks, which include input layer, hidden layer, and output layer. The hidden layer can contain one or more layers [12]. The classical BP neural network's structure is shown in Figure 1.

$V$ and $W$ are linking weighted matrices. Input layer mainly takes charge of receiving various fault messages, and every unit manifests a fault characteristic parameter. The 
main functions of hidden layer are learning the fault message of input layer and saving threshold and linking weighted value which make network approximation. Output layer mainly exports the result of diagnosis, which uses Sigmoid or hard-approximation function usually and the number of neurons is decided by the kinds of faults [13].

3.2. Establishment of the Belief Rule Base. Suppose $U=\left\{U_{i}\right.$; $i=1,2, \ldots, T\}$ is the set of rule premise attribute, and $A_{i}=$ $\left\{A_{i j} ; j=1,2, \ldots, J_{i}=\left|A_{i}\right|\right\}(i=1,2, \ldots, T)$ is the reference value set of the premise attribute $U_{i}$; then, the form of belief rule is as follows.

$$
\begin{aligned}
& \text { If } U_{1} \text { is } A_{1 n_{1}} \text { and } U_{2} \text { is } A_{2 n_{2}} \text { and so on and is } A_{T n_{T}} \text {, then } \\
& U_{T} \cdots\left\{\left(D_{1}, \overline{\beta_{1 k}}\right)\left(D_{2}, \overline{\beta_{2 k}}\right), \ldots,\left(D_{N}, \overline{\beta_{N k}}\right)\right\} \\
& \qquad(k=1,2, \ldots, L)
\end{aligned}
$$

where $A_{i n_{i}} \in A_{i}\left(n_{i}=1,2, \ldots, J_{i}\right)$ is the reference value $i$, $\overline{\beta_{i k}}(i=1,2, \ldots, N)$ is the result, the degree of confidence belonging to the output reference value $D_{i}$ meets $\sum_{i=1}^{N} \overline{\beta_{i k}} \leq 1$, and $L$ is the number of rules in rule base.

The activated weight of rule $K$ is

$$
\begin{aligned}
\omega_{k} & =\frac{\theta_{k} \alpha_{k}}{\sum_{i=1}^{L} \theta_{i} \alpha_{i}}, \\
\alpha_{k} & =\prod_{i=1}^{T_{k}}\left(\alpha_{k}^{i}\right)^{\overline{\delta_{k i}}}, \\
\overline{\delta_{k i}} & =\frac{\delta_{k i}}{\max _{i=1,2, \ldots, T_{k}}\left\{\delta_{k i}\right\}},
\end{aligned}
$$

where $\theta_{k}$ is the relative weight of rule $k, \alpha_{k}^{i}$ is the match degree of input $i$ to reference value set $A_{i}$ in rule $k, \delta_{k i}$ is the relative weight of $i$ in rule $k, a_{i}$ is the overall compatibility of input variable relative to the premise attribute $U$ in rule $i$, and $\theta_{i}$ is the weight of rule $i$.

If $\omega_{k}>0$, the rule $k$ is activated, otherwise not activated.

Because the belief degree, weight, promise attribute, and reference value are given by initial expert knowledge, the error is easy to be produced. So the generation processes of the rule base need to be optimized. The processes are as follows.

Step 1. Determine the rule belief degree, weight, premise attribute, and output result based on initial expert knowledge, and establish the initial belief rule base.

Step 2. Convert the input data into distributed form.

Step 3. Forward inference to get the simulation output based on the belief rule base and input data, and then calculate the error.

Step 4. If the error is within the allowable range, end the training optimization. Otherwise, turn to Step 5.

Step 5. According to the training data, use the FMINCON function in the MATLAB to achieve the optimization of the

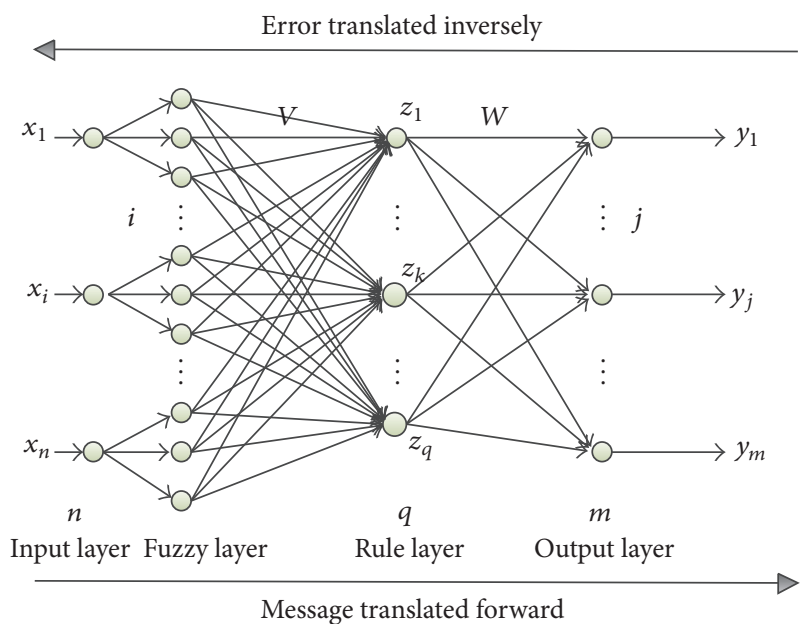

FIGURE 2: The structure of fuzzy neural network.

training parameters, save the optimization results, update the belief rule base, and then turn to Step 3 .

3.3. Structure of Improved Fuzzy Neural Network. In order to take advantage of existing experienced knowledge, we use fuzzy logic to reform neural network. The construct fuzzy neural network is constructed according to expert language rule. A 4-layer fuzzy neural network is formed by input layer, fuzzy layer, rule layer, and output layer. The structure is shown in Figure 2.

The description of every node's function is as follows.

(1) Input Layer. Input variable is original fault sign one, and the number of nodes is the number of sign variable.

(2) Fuzzy Layer. The number of nodes is the sum of all fuzzy intervals of sign variable. The number of fuzzy logic and initial membership functions is defined according to the distribution condition of every fuzzy variable and the significance of each fuzzy variable to system. Then the normalized difference variable is translated to many fuzzy sets. Then each neuron of every layer is correspondence to a fuzzy set. Its output manifests the membership to this fuzzy set.

(3) Rule Layer. Every node corresponds to a fuzzy control rule, the prerequisite of completing a fuzzy logic rule is matching operation, and the input is membership and the output is matching degree.

(4) Output Layer. According to the sum of value of fuzzy logic value and linking weight, the output is acquired.

There are three kinds of adjustable variables: one is the weighted coefficient of third and fourth layer, which manifests rule parameter; the second and third are mean value and standard deviation of the function.

3.4. Confirmation of Improved Neutral Network Training Parameters. The training and learning of neural network are mainly adjustment of interlayer linking weighted value and the core and width of affiliate function. 
TABLE 1: Training samples table.

\begin{tabular}{|c|c|c|c|c|c|c|c|c|c|c|c|}
\hline Series number & Model & $\bar{E}_{30}$ & $\bar{E}_{31}$ & $\bar{E}_{32}$ & $\bar{E}_{33}$ & $\bar{E}_{34}$ & $\bar{E}_{35}$ & $\bar{E}_{36}$ & $\bar{E}_{37}$ & $\widehat{g}_{3}$ & $\widehat{g}_{4}$ \\
\hline 1 & \multirow{4}{*}{ Imbalance } & 0.0509 & 0.522 & 0.450 & 0.538 & 0.036 & 0.505 & 0.169 & 0.152 & 0.231 & 0.572 \\
\hline 2 & & 0.727 & 0.643 & 0.639 & 0.780 & 0.057 & 0.602 & 0.113 & 0.021 & 0.154 & 0.263 \\
\hline 3 & & 0.318 & 0.358 & 0.278 & 0.415 & 0.028 & 0.334 & 0.084 & 0.245 & 0.125 & 0.241 \\
\hline 4 & & 0.857 & 0.533 & 0.538 & 0.757 & 0.066 & 0.549 & 0.100 & 0.364 & 0.265 & 0.452 \\
\hline 5 & \multirow{4}{*}{ Misaligned } & 0.293 & 0.185 & 0.147 & 0.155 & 0.018 & 0.182 & 0.380 & 0.285 & 0.341 & 0.214 \\
\hline 6 & & 0.533 & 0.520 & 0.370 & 0.487 & 0.052 & 0.548 & 0.924 & 0.214 & 0.741 & 0.285 \\
\hline 7 & & 0.166 & 0.171 & 0.131 & 0.172 & 0.020 & 0.189 & 0.298 & 0.623 & 0.321 & 0.241 \\
\hline 8 & & 0.467 & 0.247 & 0.143 & 0.272 & 0.042 & 0.277 & 0.653 & 0.214 & 0.854 & 0.365 \\
\hline 9 & \multirow{4}{*}{ Looseness } & 0.304 & 0.058 & 0.051 & 0.136 & 0.062 & 0.156 & 0.072 & 0.247 & 0.280 & 0.157 \\
\hline 10 & & 0.617 & 0.124 & 0.197 & 0.257 & 0.107 & 0.222 & 0.090 & 0.352 & 0.264 & 0.514 \\
\hline 11 & & 0.302 & 0.042 & 0.077 & 0.114 & 0.058 & 0.110 & 0.046 & 0.452 & 0.153 & 0.214 \\
\hline 12 & & 0.667 & 0.075 & 0.127 & 0.214 & 0.106 & 0.161 & 0.059 & 0.632 & 0.352 & 0.241 \\
\hline 13 & \multirow{4}{*}{ Rub-impact } & 0.108 & 0.286 & 0.245 & 0.161 & 0.053 & 0.211 & 0.020 & 0.214 & 0.152 & 0.741 \\
\hline 14 & & 0.302 & 0.806 & 0.595 & 0.614 & 0.095 & 0.732 & 0.102 & 0.145 & 0.215 & 0.541 \\
\hline 15 & & 0.120 & 0.428 & 0.246 & 0.352 & 0.045 & 0.402 & 0.063 & 0.362 & 0.384 & 0.562 \\
\hline 16 & & 0.297 & 0.001 & 0.023 & 0.076 & 0.053 & 0.057 & 0.026 & 0.264 & 0.274 & 0.247 \\
\hline
\end{tabular}

TABLE 2: Testing samples.

\begin{tabular}{lccccccccccc}
\hline Serial number & $\bar{E}_{30}$ & $\bar{E}_{31}$ & $\bar{E}_{32}$ & $\bar{E}_{33}$ & $\bar{E}_{34}$ & $\bar{E}_{35}$ & $\bar{E}_{36}$ & $\bar{E}_{37}$ & $\widehat{g}_{3}$ & $\widehat{g}_{4}$ \\
\hline 1 & 1.181 & 0.821 & 0.708 & 0.881 & 0.068 & 0.955 & 0.228 & 0.245 & 0.365 & 0.241 \\
2 & 0.9 & 0.461 & 0.357 & 0.341 & 0.040 & 0.44 & 1.055 & 0.258 & 0.241 & 0.854 \\
3 & 0.798 & 0.238 & 0.209 & 0.364 & 0.188 & 0.382 & 0.159 & 0.218 & 0.368 & 0.247 \\
4 & 0.309 & 0.547 & 0.465 & 0.292 & 0.107 & 0.401 & 0.026 & 0.285 & 0.421 & 0.548 \\
\hline
\end{tabular}

TABLE 3: Output result.

\begin{tabular}{|c|c|c|c|c|c|}
\hline Serial number & $d_{1}$ & $d_{2}$ & $d_{3}$ & $d_{4}$ & Expected output \\
\hline 1 & 1.0021 & 0.0035 & 0.0106 & 0.0202 & 1000 \\
\hline 2 & 0.0180 & 1.0142 & 0.0240 & 0.0012 & 0100 \\
\hline 3 & 0.0028 & 0.0032 & 1.0421 & 0.0507 & 0010 \\
\hline 4 & 0.0109 & 0.0207 & 0.0253 & 1.0437 & 0001 \\
\hline
\end{tabular}

(1) Linking Weighted Value. Between fuzzy layer and rule layer, the value is 1 while linking and 0 while not linking. We only train the linking weighted value between rule layer and output layer.

(2) Confirmation of Affiliate Function. The input data need to be normalized difference because of the characteristic of neural network and the mainly transform function. While BP network is applied into solving problem, the condition of convergence slow or little always appears. A good way to normalize difference can have an improvement on the property of network. In this paper, fuzzy membership normalized difference is used. Because the relationship between fault and sign is fuzzy, whether fault exists does not have distinct bounds and the fault obeys the distribution of some membership function. In a word, the kind of membership we choose is as follows: the value of membership function increases in some method with the increase of independent variable. The expression is

$$
\mu(x)=\frac{k x^{2}}{1+k x^{2}}
$$

And ensured self-Cauchy membership function satisfies the above demand.

(3) Learning Velocity (Step-Size) $l_{r}$. Set the initial value as 0.01 , and it can be satisfied according to the following formula:

$$
l_{r}= \begin{cases}1.05 l_{r}, & E(k)<E(k-1), \\ 0.7 l_{r}, & E(k)>E(k-1) \cdot 1.04 \\ l_{r}, & \text { others. }\end{cases}
$$

(4) Momentum Factor mc. Set the initial value as 0.95 , and it can be satisfied according to the following formula:

$$
l_{r}= \begin{cases}0, & E(k)<E(k-1), \\ 0.95, & E(k)>E(k-1) \cdot 1.04, \\ \mathrm{mc}, & \text { others. }\end{cases}
$$

(5) Error. It is a very small number and can be ascertained according to specific condition. 
TABLE 4: Two-mixed fault training.

\begin{tabular}{lcccccccccc}
\hline Serial number & $\bar{E}_{30}$ & $\bar{E}_{31}$ & $\bar{E}_{32}$ & $\bar{E}_{33}$ & $\bar{E}_{34}$ & $\bar{E}_{35}$ & $\bar{E}_{36}$ & $\bar{E}_{37}$ & $\widehat{g}_{3}$ & $\widehat{g}_{4}$ \\
\hline 1 & 0.0809 & 0.622 & 0.550 & 0.468 & 0.236 & 0.305 & 0.469 & 0.352 & 0.431 & 0.672 \\
2 & 0.127 & 0.543 & 0.439 & 0.560 & 0.347 & 0.302 & 0.513 & 0.421 & 0.514 & 0.663 \\
\hline 5 & 0.523 & 0.432 & 0.642 & 0.334 & 0.676 & 0.184 & 0.295 & 0.693 & 0.073 & 0.114 \\
6 & 0.518 & 0.398 & 0.579 & 0.299 & 0.702 & 0.165 & 0.249 & 0.703 & 0.081 & 0.125 \\
\hline
\end{tabular}

TABLE 5: Training result.

\begin{tabular}{lcccccr}
\hline Serial number & $d_{1}$ & $d_{2}$ & $d_{3}$ & $d_{4}$ & Expected output & Model \\
\hline 1 & 1.0034 & 0.9924 & 0.0305 & 0.0043 & 1100 & Imbalance and misaligned fault \\
2 & 1.0200 & 1.0324 & 0.0130 & 0.0031 & 1100 & Looseness and rub-impact fault \\
\hline 3 & 0.0028 & 0.0032 & 1.0421 & 0.9507 & 0011 & 0011 \\
\hline
\end{tabular}

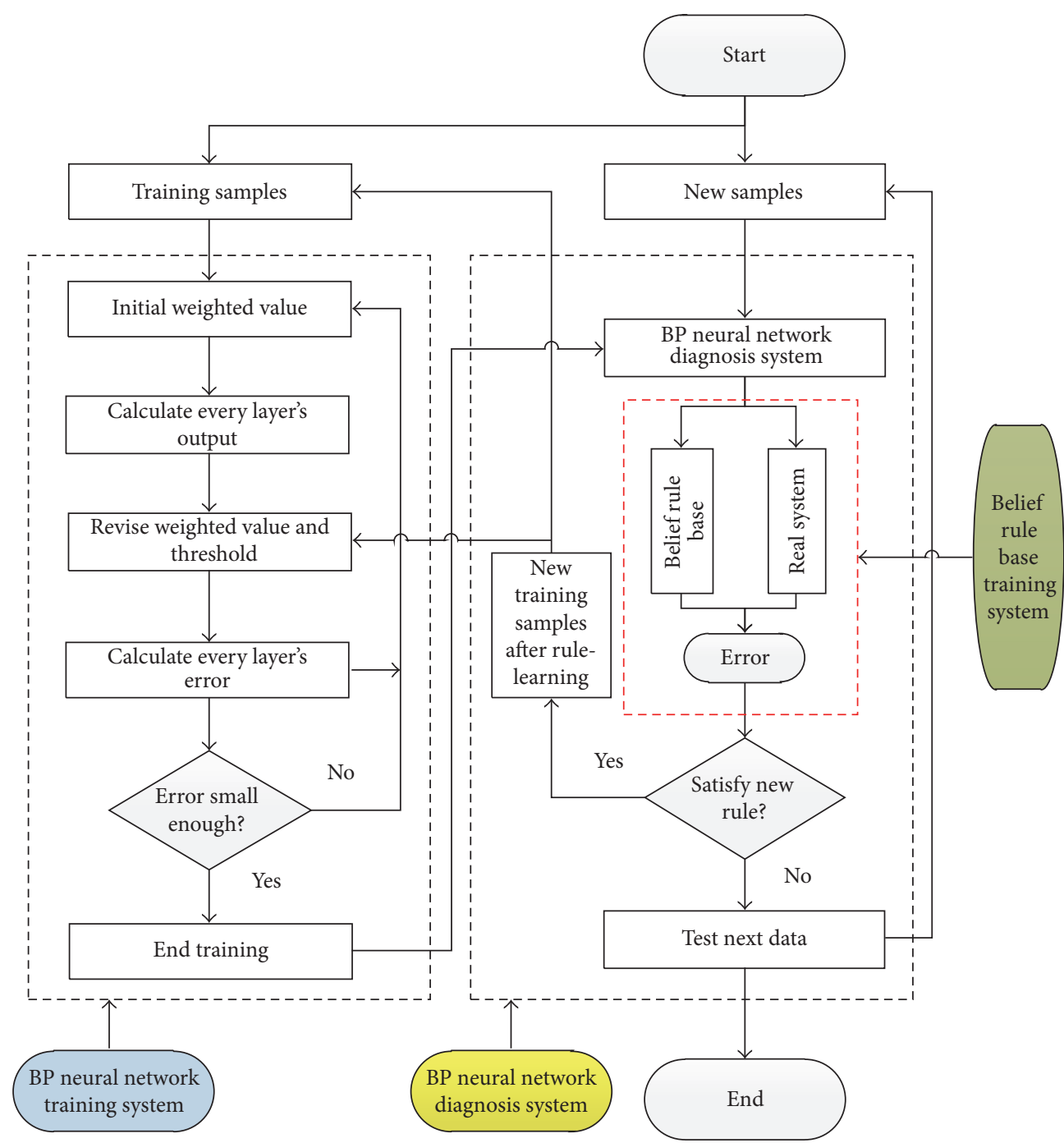

FIGURE 3: Procedure of rule-learning base BP neural network fault diagnosis. 

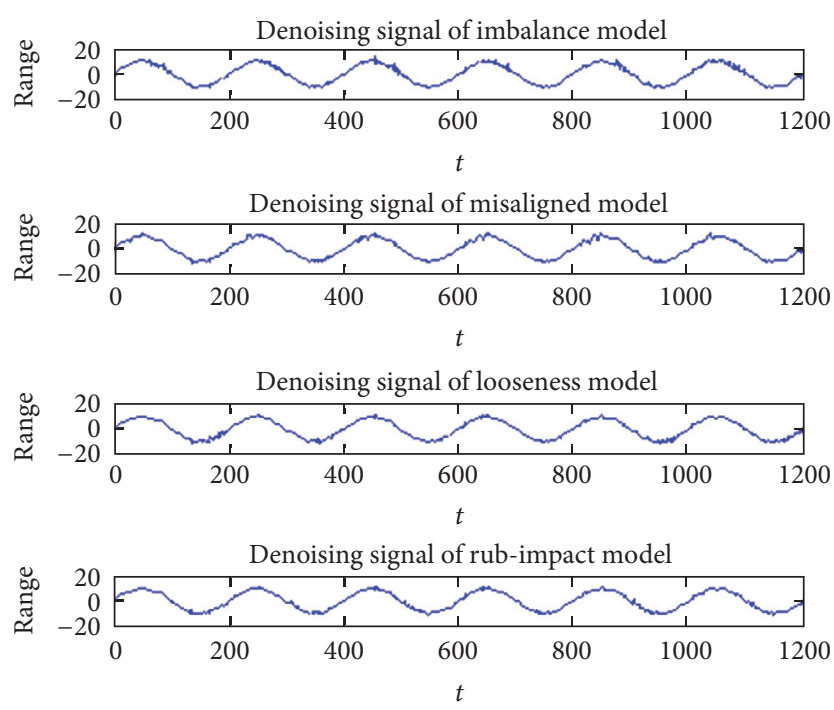

FIgURE 4: Denoise effect on figure of sampling signal.

(6) Maximum Number of Network's Training Processes. It is one of the end conditions of the network training. Stop it when it does not converge until maximum number, which manifests that the property of convergence is bad or the maximum number of training processes is too small.

\subsection{The Procedure of BP Neural Network Fault Diagnosis Based on Rule-Learning}

Step 1. Classify samples into training samples and test (new) samples.

Step 2. Construct initial fuzzy neural network training system based on Section 3.4 and form improved fuzzy neural network diagnosis system after several training processes.

Step 3. Diagnosis is done by the test samples of trained BP neural network diagnosis system, and judge whether the fault is a new fault or not. A new sample is formed if it is a new fault. We train the improved BP neural network and realize the function of self-learning.

\section{Simulation and Test}

4.1. Description of Problem. We put rolling bearing as the fault diagnosis target and draw its vibration signal. The inputs are the 8 frequencies of time and frequency territory's power and sharpness, which manifest by $S_{1}-S_{10}$. The 10 characteristics are condition attributes $C=\left\{S_{1}, S_{2}, \ldots, S_{10}\right\}$. The rolling bearing has five states: formal, imbalance, misalignment, rubimpact, and looseness, which are manifested by $(0,0,0,0)$, $(1,0,0,0),(0,1,0,0),(0,0,1,0)$, and $(0,0,0,1)$.

4.2. Sole Fault Simulation. It is done through the experiment bench to sample and denoise the signal as shown in Figure 4.

The training samples are shown in Table 1 after analysing and drawing.

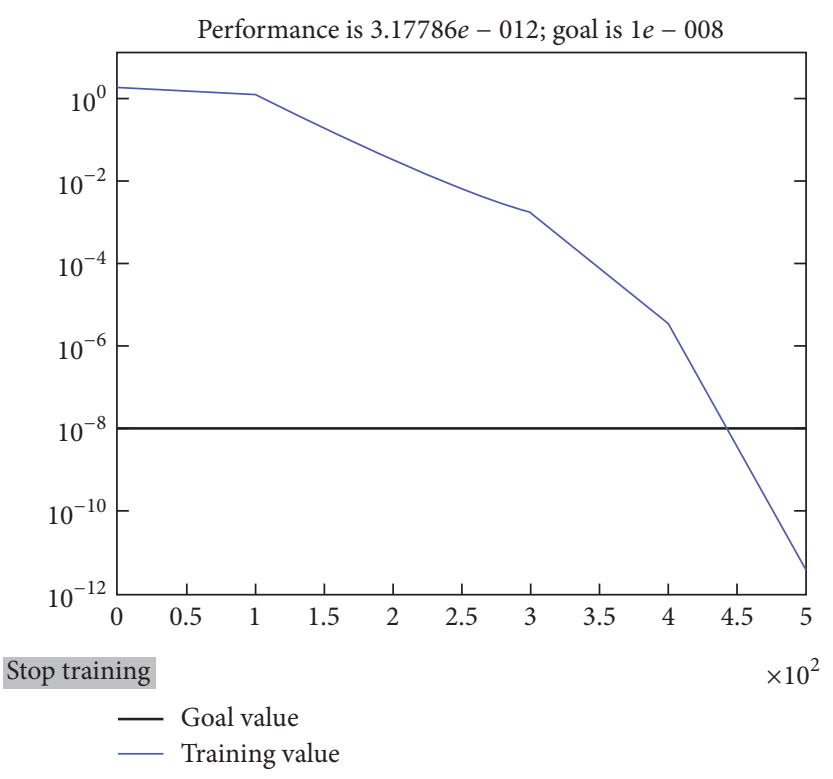

FIGURE 5: Procedure of training.

The BP neural network has 10 input nodes, 4 output nodes, and 21 hidden nodes. It uses the learning algorithm of Section 3.4. The maximum training processes number is 1000 . The precision of training is $1 e-8$ and learning efficiency is 0.01 . The 16 groups of Table 1 are used to train. The procedure is shown in Figure 5.

We can see from Figure 5 that the error request after 450 steps is good and the convergence effect is very good.

In order to test the effect of BP neural network fault diagnosis system after 16 samples training, we acquire 4 test samples in Table 2. The test results are shown in Table 3.

We can conclude from Table 3 that the outputs of 4 test samples coincide with expected value. The accuracy of model is tested.

4.3. Mixed Fault Simulation. The mixed fault is common in the real fault. We set two manual mixed faults as in Table 4.

The samples of Table 4 cannot be distinguished by the BP neural network fault diagnosis system trained by Table 1 . But it can acquire the result of Table 5 by the belief rule base of Figure 3.

We train the BP neural network again by using the data of Table 5 as a new training sample. Comparing the expected output and the actual output in Table 5, it can be found that the neural network trained by belief rule base has the diagnosis ability to these two kinds of mixed fault.

\section{Conclusion}

Because of the distribution of training sample, the veracity of training result of neural network is hard to ensure by only using neural network to diagnose fault. A neural network fault diagnosis based on rule base is promoted, which transfers expert's experience to rule by fuzzy process and then applies to neural network. This method ensures that 
the training sample can respond and the veracity of neural network fault diagnosis is improved.

\section{Competing Interests}

There are no competing interests related to this paper.

\section{References}

[1] D.-H. Zhou and Y.-Y. Hu, "Fault diagnosis techniques for dynamic systems," Acta Automatica Sinica, vol. 35, no. 6, pp. 748-758, 2009.

[2] S. X. Ding, Model-Based Fault Diagnosis Techniques: Design Schemes, Algorithms and Tools, Springer, London, UK, 2nd edition, 2013.

[3] A. Haghani Abandan Sari, Data-driven design of fault diagnosis systems, Springer, Wiesbaden, Germany, 2014.

[4] L. Qin, X. He, and D. Zhou, "A survey of fault diagnosis for swarm systems," Systems Science \& Control Engineering, vol. 2, no. 1, pp. 13-23, 2014.

[5] C. Chen, B. Bai, and H. Yu, "Improved algorithm for threelayer percetrons and its application to fault diagnosis," Journal of Vibration, Measurement \& Diagnosis, vol. 20, no. 1, pp. 19-24, 2000.

[6] S. Ergezinger and E. Thomsen, "An accelerated learning algorithm for multilayer perceptrons: optimization layer by layer," IEEE Transactions on Neural Networks, vol. 6, no. 1, pp. 31-42, 1995.

[7] K. Yuan and M. Liu, "The application of neural network in fault diagnosis inference," Journal of Shandong Institute of Commerce and Technology, no. 6, pp. 97-100, 2006.

[8] B. Liu, C. Sun, and X. Wang, "The neural network methodology for solid rocket motor fault diagnosis," Computer Simulation, no. 25, pp. 47-50, 2008.

[9] Y. Zhang and J. Wei, "Software fault predicting system and its knowledge model library," Radar \& ECM, no. 3, pp. 60-63, 2007.

[10] J. Wu and P. Deng, "A fault diagnosis expert system based on regular complex artificial neural network," Journal of Hubei Engineering University, no. 26, pp. 32-35, 2006.

[11] J. Wu and J. Xiao, Intellectual Fault Diagnosis and Expert System, Science Press, Beijing, China, 1997.

[12] S. V. Kamarthi and S. Pittner, "Accelerating neural network training using weight extrapolations," Neural Networks, vol. 12, no. 9, pp. 1285-1299, 1999.

[13] Fly Science and Technology Product Research and Development Center, Neural Network Theory and Realization of Malab7, Publishing House of Electronics Industry, Beijing, China, 2005. 


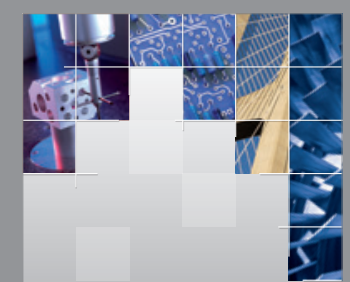

\section{Enfincering}
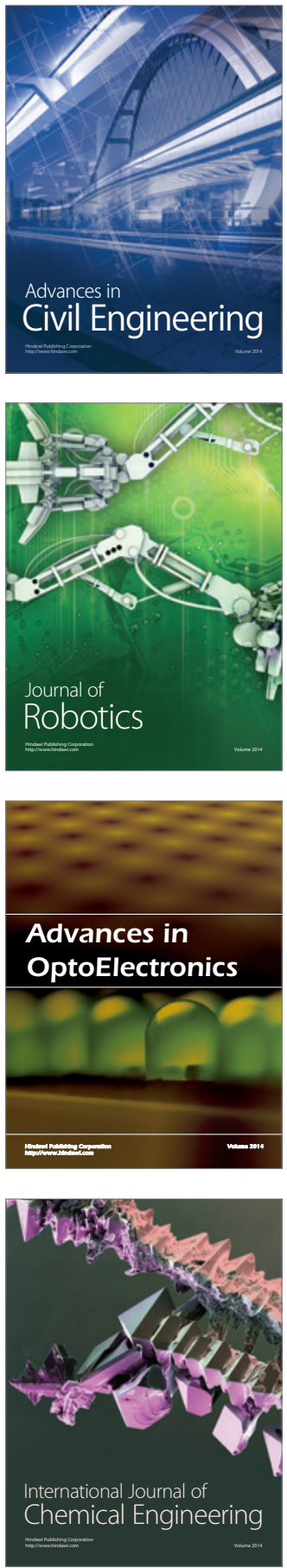

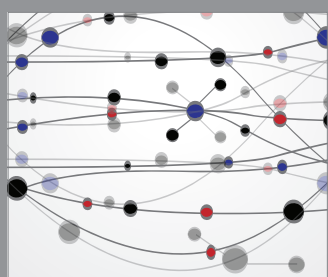

The Scientific World Journal

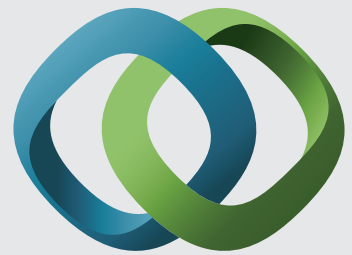

\section{Hindawi}

Submit your manuscripts at

https://www.hindawi.com
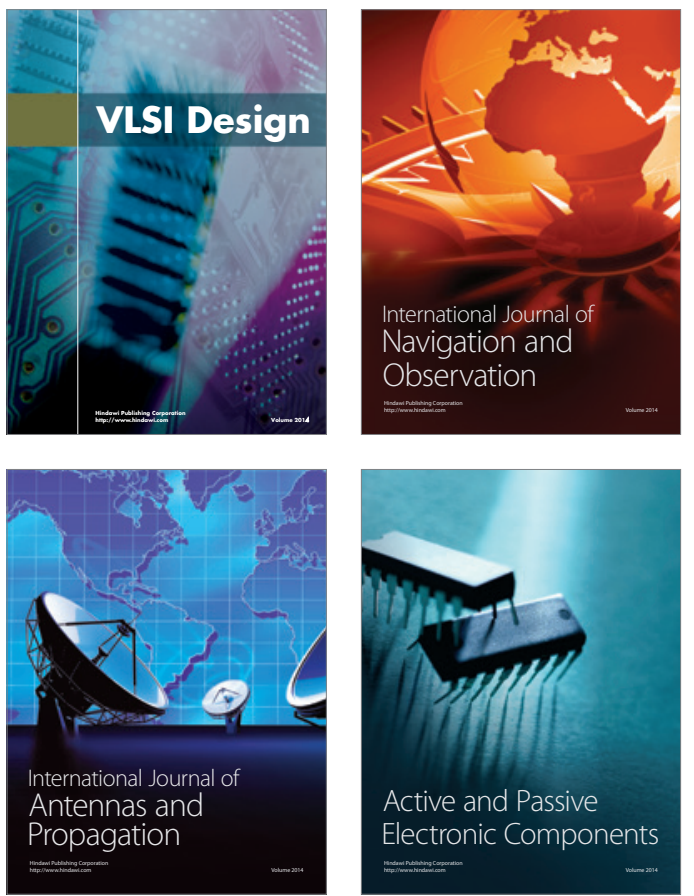
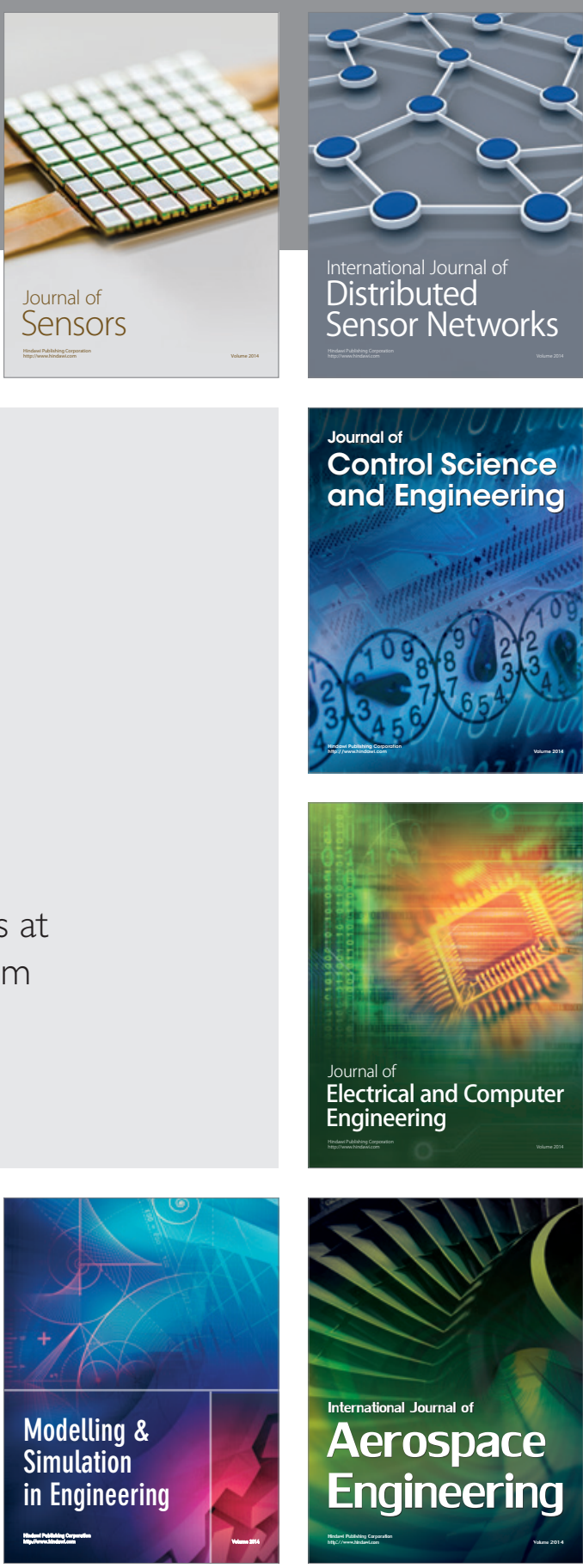

International Journal of

Distributed

Sensor Networks

$-$

Joumal of

Control Science

and Engineering
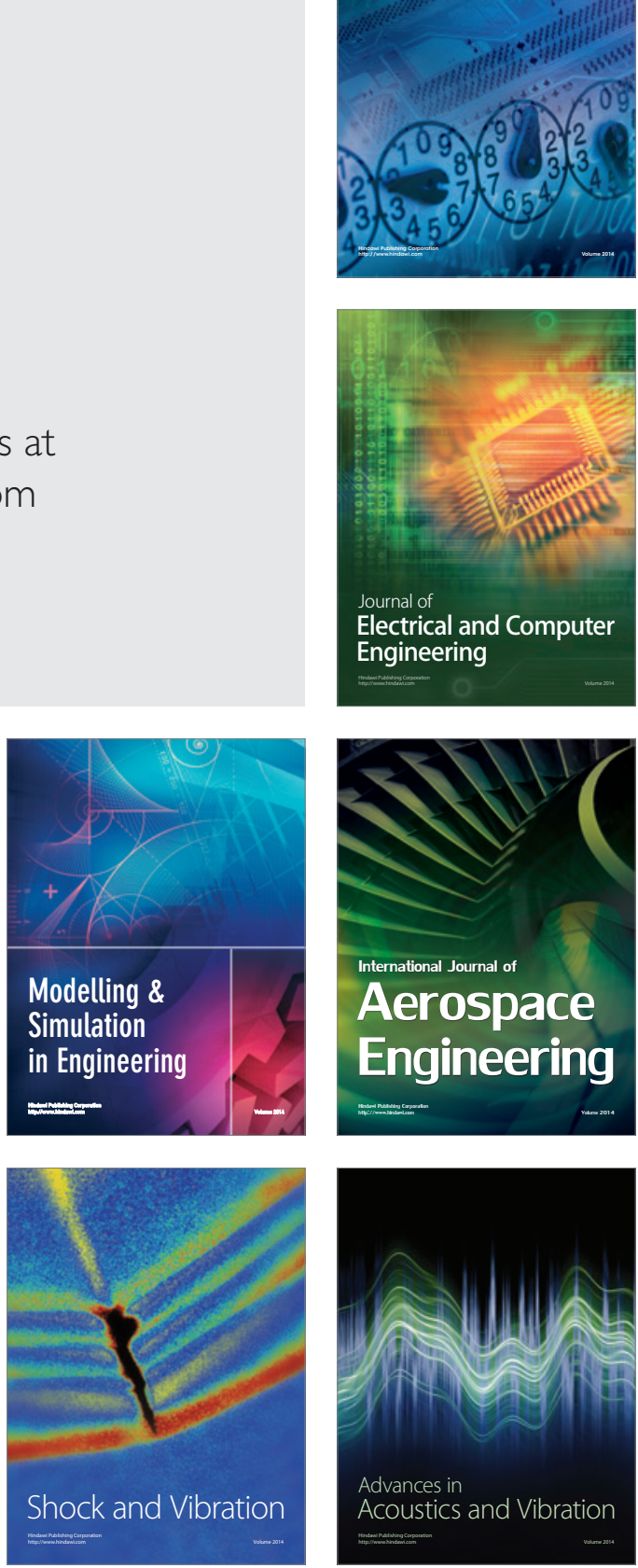